OPEN ACCESS

Edited by:

Jean-Francois Ripoll,

CEA DAM Île-de-France, France

Reviewed by:

Theodore Sarris,

Democritus University of Thrace,

Greece

Clare Watt,

Northumbria University,

United Kingdom

*Correspondence:

Solène Lejosne

solene@berkeley.edu

Specialty section: This article was submitted to

Space Physics,

a section of the journal Frontiers in Astronomy and Space

Sciences

Received: 28 November 2021

Accepted: 13 January 2022

Published: 17 February 2022

Citation:

Lejosne S, Fejer BG, Maruyama N and Scherliess L (2022) Radial Transport of

Energetic Electrons as Determined From the "Zebra Stripes" Measured in the Earth's Inner Belt and Slot Region. Front. Astron. Space Sci. 9:823695. doi: 10.3389/fspas.2022.823695

\section{Radial Transport of Energetic Electrons as Determined From the "Zebra Stripes" Measured in the Earth's Inner Belt and Slot Region}

\author{
Solène Lejosne ${ }^{1 *}$, Bela G. Fejer ${ }^{2}$, Naomi Maruyama ${ }^{3}$ and Ludger Scherliess ${ }^{2}$ \\ ${ }^{1}$ Space Sciences Laboratory, University of California, Berkeley, Berkeley, CA, United States, ${ }^{2}$ Center for Atmospheric and Space \\ Sciences, Utah State University, Logan, UT, United States, ${ }^{3}$ Laboratory for Atmospheric and Space Physics, University of \\ Boulder, Boulder, CO, United States
}

The "zebra stripes" are drift-periodic structures present in the form of peaks and valleys in energetic (tens to hundreds of keV) electron spectrograms in the Earth's inner belt and slot region. Their characteristics inform of preceding electric field disturbances. Specifically, their amplitude contains information on the radial transport of trapped particles generated by azimuthal electric field disturbances. We introduce a method to quantify radial transport from the measured amplitude of the zebra stripes, and we apply it to the zebra stripes observed on 16 February 2014. The findings are compared with results from a particle tracing code that leverages an empirical analytical model for the electric field disturbances. The measured amplitude of the zebra stripes indicates that the electric field disturbances transported the trapped population coherently, over radial distances spanning several hundreds to thousands of kilometers. The magnitude of radial transport is shown to depend on angular drift frequency and initial location (equatorial radial distance and magnetic local time) in the disturbance. The model-observation comparison suggests that the timing for electric field variations provided by the model is valid for studying radial transport in the inner belt and slot region. On the other hand, we found discrepancies when comparing radial transport magnitudes. When assuming that radial transport varies as $L^{3}$, as weakly suggested by the data for set angular drift frequencies, and extrapolating observations to $L=1$, the amount of experimental transport obtained is two to three times greater than numerical estimates. Outputs from the standalone version of the Rice Convection Model (RCM) suggests that the electric field disturbances are likely greater than the estimates provided by the empirical model. RCM also supports the idea that radial transport driven by the prompt penetration of magnetospheric convection varies as $L^{3}$ in the inner belt and slot region.

Keywords: radial transport, prompt penetration electric fields, disturbance electric fields, trapped particles, Earth's inner radiation belt, van allen probes 


\section{INTRODUCTION}

The Van Allen Probes mission (Mauk et al., 2013) has provided large amounts of measurements of the zebra stripes below an equatorial altitude of about three Earth Radii $\left(\mathrm{L}<3\right.$, i.e. $<54^{\circ}$ in magnetic latitude for the field line footpoint). The zebra stripes are drift-periodic structures in the form of well-defined peaks and valleys in the spectrograms of energetic (tens to hundreds of $\mathrm{keV}$ ) electrons. They are usually present over continuous portions (several Earth radii) of the inner belt and slot region (Imhof and Smith, 1965; Ukhorskiy et al., 2014). Similar structures have also been detected in the inner magnetosphere of Saturn, where they were used to provide information on magnetospheric convection (Sun et al., 2021). Indeed, zebra stripe generation mechanisms usually assume that some spatial and/or temporal variations of the magnetospheric electric field modify trapped particles' drift motion depending on drift phase (e.g. Sauvaud et al., 2013; Ukhorskiy et al., 2014; Lejosne and Roederer, 2016; Liu et al., 2016; Selesnick et al., 2016).

One of the feature of the zebra stripes is that the drift frequency separation between successive stripes decreases with time (Lejosne and Roederer, 2016; Liu et al., 2016). This characteristic has been leveraged recently to demonstrate that the time at which zebra stripes are generated is usually associated with substorm onset (Lejosne and Mozer, 2020a). As a result, the zebra stripes are now thought to be routinely generated by the magnetospheric electric field disturbances occurring around substorm onset. To modify drift motion depending on drift phase, the electric field disturbance needs to vary rapidly enough to violate the third adiabatic invariant of trapped energetic electrons. In other words, it needs to have a characteristic timescale shorter than a couple of hours (that is, the drift period timescale).

Magnetospheric electric fields and conjugate ionospheric electric fields are usually related under the assumption of equipotential field lines, as is the case within the plasmasphere (e.g. Mozer, 1970; Lejosne and Mozer, 2016). Ionospheric electric field disturbances associated with magnetic activity have been well studied experimentally (e.g. Fejer et al., 2017 and references therein). They are usually viewed as the combination of two processes: 1) a large, sudden and relatively short-lived (<a couple of hours) perturbation associated with the prompt penetration of high-latitude electric fields and 2) a perturbation occurring on a slower timescale due to the dynamo action of storm time winds. In that context, the zebra stripes are now thought to be a mark of the radial transport associated with the prompt penetration of magnetospheric convection.

Radial transport in the absence of significant magnetic field perturbations is primarily due to time variations of the azimuthal component of the electric field (e.g. Fälthammar, 1965). This component has been most accurately measured at the geomagnetic equator in the ionosphere, where the magnetic field lines are typically aligned in the north-south direction and the vertical ion drift can be easily converted into a zonal electric field. Years of radar observations of the ionospheric drift resulted in a relatively simple analytical model for the storm time dynamics of the disturbance electric fields over Jicamarca, Peru (Fejer and Scherliess, 1997). This analytical model is leveraged in the following.
The objective of this study is to demonstrate that the zebra stripes contain information on important, yet mostly uncharted drivers of the Earth's inner radiation belt and slot region. Namely, we show that the zebra stripes contain information: 1) on the dynamics of electric field disturbances, and 2) on the irreversible radial transport of trapped populations. Observational and modeling resources are introduced in Section 2. In particular, we describe the analytical empirical model for electric field disturbances over Jicamarca. We also detail how to quantify radial transport from measured fluctuations in trapped particles' directional differential fluxes in Section 2. We apply the method in Section 3. We show that the analysis of the zebra stripes of 16 February 2014 suggests radial transport up to a few thousands of kilometers, variable as a function of drift frequency and initial location, yet consistent over a broad range of $L$ values $(\Delta \mathrm{L}>1)$. We leverage the model for ionospheric electric field disturbances at the equator to compute radial transport of notionally trapped particles at $\mathrm{L}=1$. A comparison between model and observation is presented in Section 3, and it is discussed in Section 4 in the light of additional results from the Rice Convection Model (RCM).

\section{MATERIALS AND METHODS}

We present the system of equations relative to drift motion and radial transport for trapped energetic particles (Section 2.1) and the empirical electric field model chosen to solve them (Section 2.2). Both elements are used in a particle tracing code, whose outputs are compared with experimental estimates. In Section 2.3, we present the equation to implement in order to determine radial transport from measured fluctuations in directional differential fluxes. The assumptions underlying the theoretical framework are also provided in Section 2.3.

\subsection{Trapped Particles' Drift Motion in the Inner Belt and Slot Region}

The objective of this Section is to present the theoretical framework necessary to describe drift motion and radial transport in the inner belt and slot region.

\subsubsection{Trapped Particles' Azimuthal Drift Motion}

In the absence of significant time variation of the magnetic and electric fields, trapped energetic electrons drift eastward around the Earth, along closed surfaces known as drift shells. There is on average no radial transport as they remain at about the same equatorial distance from the Earth. The frequency of this periodic drift motion, $\Omega / 2 \pi$, is the sum of a relatively small contribution due to the action of the electric field, $\Omega_{E} / 2 \pi$, and a main magnetic contribution, $\Omega_{B} / 2 \pi$ :

$$
\Omega=\Omega_{E}+\Omega_{B}
$$

The electric drift frequency is mostly constant and equal to corotation frequency $\left(\Omega_{E}=2 \pi / 24 \sim 0.26 \mathrm{rad} / \mathrm{hr}\right)$. It can slightly $(\sim 10 \%)$ decrease during active times (e.g. Sandel et al., 2003; Lejosne and Mozer, 2018). On the other hand, the magnetic drift 


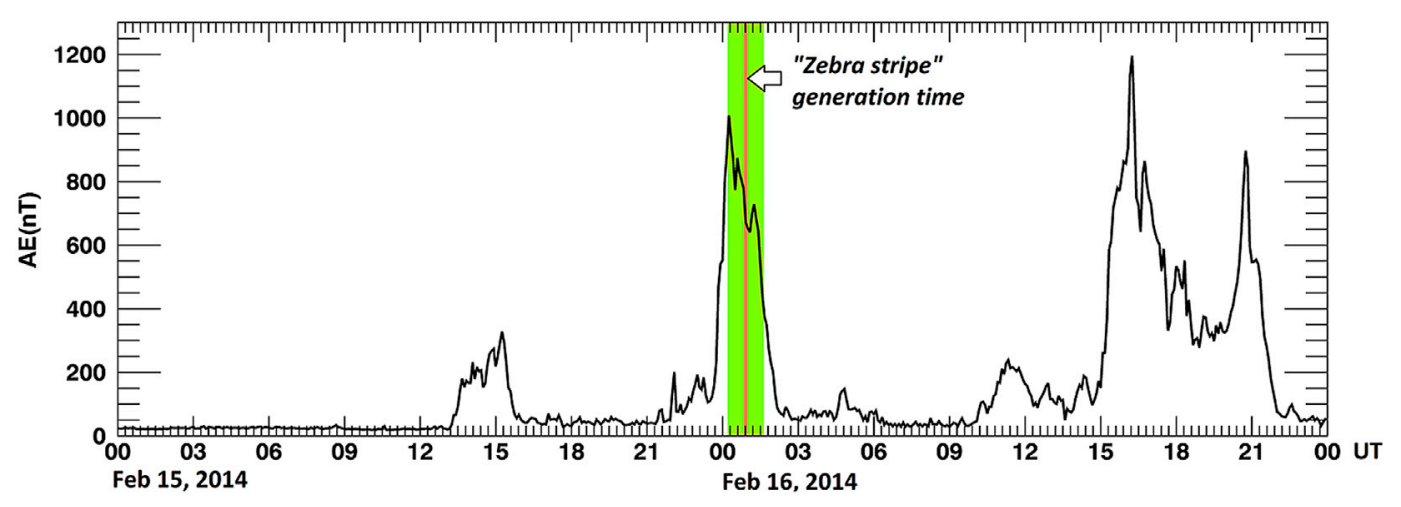

FIGURE 1 | The time evolution of the AE index on 15 February 2014 and 16 February 2014. The estimated time for the generation of zebra stripes is indicated by an orange line. The standard deviation of the estimate is shaded in green. A substorm onset occurred during this time interval, at 00:15 UT on 16 February 2014 according to the criteria set by Newell and Gjerloev (2011).

frequency is a function of the magnetic field topology (magnitude, gradient, and curvature of the magnetic field). The magnetic drift frequency also depends on the particles' characteristics (kinetic energy, charge, and to a lesser extent, pitch angle). In the case of electrons trapped in a magnetic dipole field:

$$
\Omega_{B}(T, L, y)=\frac{3 L T}{e B_{E} R_{E}^{2}}\left(\frac{\gamma+1}{\gamma}\right) \frac{\mathcal{D}(y)}{\mathcal{T}(y)}
$$

Where $T$ is the kinetic energy, $\gamma$ is the Lorentz factor, $R_{E}=$ $6400 \mathrm{~km}$ is one Earth radius, $L$ is the normalized equatorial radial distance, $L=r / R_{E}, B_{E}=30,000 \mathrm{nT}$ is the magnetic equatorial field at the surface of the Earth, $e$ is the elementary charge, and $\mathcal{D}$ and $\mathcal{T}$ are functions of the sine of the equatorial pitch angle, $y$. The ratio $\mathcal{D}(y) / T(y)$ lies between $1 / 3$ and $1 / 2$. It is equal to $1 / 2$ for equatorial particles $(y=1)$ and it decreases monotonically with decreasing pitch angle (Schulz and Lanzerotti, 1974; see also Lejosne and Mozer, 2020a, 2020b for the definitions of $\mathcal{D}$ and $\mathcal{T}$ ).

The value of the sine of the equatorial pitch angle, $y$, is related to the pitch angle measured along the same field line, $\alpha$, through conservation of the first adiabatic invariant: $y=$ $\sin \alpha \sqrt{B_{o} / B}$ where $B$ is the local magnetic field magnitude, and $B_{o}$ is the magnetic field magnitude at the magnetic equator of the same field line. In this study, the ratios between $B$ and $B_{o}$ are calculated according to the International Geomagnetic Reference Field to which the Kpdriven Tsyganenko (1989) magnetic field model is superimposed. Because the analysis is in the Earth's inner belt and slot region $(\mathrm{L}<3)$, the choice of the magnetic field model is unimportant provided that it remains consistent with the assumption of a magnetic field that is mainly dipolar.

\subsubsection{Trapped Particles' Radial Motion}

While the magnetic field of the inner belt and slot region remains essentially equal to the internal geomagnetic field, the electric field varies with time, $t$, driving radial transport. In polar coordinates $(r, \varphi)$, the radial transport of trapped particles is described by the following set of equations of motion:

$$
\left\{\begin{array}{c}
\frac{d r}{d t}=\frac{E_{\varphi}(r, \varphi ; t)}{B_{o}} \\
\frac{d \varphi}{d t}=\Omega
\end{array}\right.
$$

where $E_{\varphi}$ is the azimuthal component of the time varying electric field at the magnetic equator, $r$ is the equatorial radial location, and $\varphi$ is the drift phase, i.e., the magnetic local time of the trapped particles. In this formulation, radial motion due to the internal geomagnetic multipoles (Roederer et al., 1973) and to the quiet time wind dynamo (Richmond et al., 1980) is assumed to be small in comparison with radial motion driven by the time-varying electric fields. The main cause of drift shell distortion for energetic particles of the inner belt, the quiet time wind dynamo, provides $6-8 \mathrm{kV}$ for the average dawnto-dusk electric potential variation at $\mathrm{L}=1.30$ (Lejosne et al., 2021), and local time dependent distortion of $\Delta L \sim 0.01$, i.e., several tens of kilometers (see also: Selesnick et al., 2016).

To solve this system of equations, information on the spatiotemporal variations of the azimuthal component of the electric field is required. We use an empirical model for the time varying electric fields of the equatorial ionosphere. It is introduced in the following (Section 2.2).

\subsection{Analytical Empirical Model for the Azimuthal Electric Field Disturbances of the Equatorial lonosphere}

A mathematical formula for the azimuthal storm time ionospheric electric field disturbances at the geomagnetic equator has been developed by Fejer and Scherliess (1995), Fejer and Scherliess (1997) leveraging 20 years (1968-1988) and nearly five thousand hours of radar measurements of the F-region vertical plasma drift (300-400 km) over Jicamarca, Peru. The formulation with the highest time resolution uses the time history of the $15 \mathrm{~min}$ averaged $\mathrm{AE}$ index to provide a description of the prompt penetration and disturbance dynamo electric fields. The magnitude of the local time dependent prompt penetration electric fields is determined by the $\mathrm{AE}$ variations over the past 
$15 \mathrm{~min}(A E(t)-A E(t-15 \mathrm{~min})), \quad$ at $30 \mathrm{~min}$ time delay $(A E(t-15 \mathrm{~min})-A E(t-45 \mathrm{~min}))$, and at $75 \mathrm{~min}$ time delay $(A E(t-45 \mathrm{~min})-A E(t-105 \mathrm{~min}))$. The electric fields generated by the disturbance wind dynamo arise from shorter term (1-12 h) and longer term (22-28 h) disturbance dynamo effects. The short-term effects are represented using two parameters $A E_{d}(1-6$ hours $)$ and $A E_{d}(7-12$ hours $)$, which denote the average value of the $\mathrm{AE}$ index above a value of $130 \mathrm{nT}$ from 1 to $6 \mathrm{~h}$ before the time $t$, and from 7 to $12 \mathrm{~h}$ prior the time $\mathrm{t}$, respectively. The longer term disturbance dynamo electric fields $A E_{d}(22-28$ hours $)$ arise when the average $\mathrm{AE}$ index from 22 to $28 \mathrm{~h}$ prior to the time $t$ is greater than $200 \mathrm{nT}$. The model informs the dynamics of the azimuthal (i.e. zonal) component of the electric field in the equatorial region of the ionosphere above the radar location $(B=25,000 n T)$. In the following, we assume that the model remains valid at the magnetic equator of $L=1(B=30,000 n T)$

On 16 February 2014, zebra stripes were noticeable below $L=3$ in the energetic electron spectrograms measured by both Van Allen Probes, for more than $10 \mathrm{~h}$ starting 05:40 UT (Liu et al., 2016). The analysis of the drift frequency separation between successive stripes suggested that the features were generated by fast electric field variations occurring at 00:55 UT $\pm 42 \mathrm{~min}$ on 16 February 2014 (Lejosne and Mozer, 2020a), a time interval that coincides with substorm onset-at 00:15 UT on 16 February 2014 according to the criteria set by Newell and Gjerloev (2011a). The concurrence between substorm onset and generation time is a characteristic feature of the zebra stripes (Lejosne and Mozer, 2020a). The time series of the Auroral Electrojet (AE) index from NASA/GSFC's OMNI data set through OMNIWeb (https://omniweb.gsfc.nasa. gov/) is provided with a 5 min time resolution in Figure 1. This time series is the input to the model for equatorial electric field disturbances. The AE index increases sharply at the beginning of 16 February $2014(+900 n T$ in less than an hour). It reaches a maximum of 1,008 nT at 00:15 UT. Then, it decreases by about $-300 \mathrm{nT}$ in 1 hour before decreasing even faster $(-740 \mathrm{nT} / \mathrm{hr})$ and reaching quiet time value levels below $100 \mathrm{nT}$ by 02:10 UT.

The mostly quiet AE values of 15 February 2014 and the fast variations measured around substorm onset indicates that the main source of electric field disturbances for this event is the prompt penetration of magnetospheric convection.

\subsection{Relating Measured Fluctuations in Trapped Particles' Directional Differential Fluxes and Radial Transport}

Sudden variations of the fields produce drift echoes (e.g., Lanzerotti et al., 1967), that is, drift-periodic fluctuations in flux measurements. The amplitude of the fluctuation is related to the radial transport for trapped populations below $L=3$ (Lejosne and Mozer, 2020b):

$$
\frac{\delta j}{j}(T, y, L)=\psi(T, y, L) \frac{d L}{L}
$$

where $\delta j=j\left(T, y, L ; t^{+}\right)-j\left(T, y, L ; t^{-}\right)$is the fluctuation in directional differential flux, $j$, and $t^{-}$and $t^{+}$are times before and after the occurrence of electric field disturbances, respectively.
The denominator, $j$, is the flux, measured at $t^{-}$. This equation is equivalent to detailing the conservation of phase space density, $f$, along the dynamical paths of particles assuming conservation of the first two adiabatic invariants. The function $\psi$ controls how radial transport, $d L$, shows up in flux measurements. It is defined as:

$$
\psi(T, y, L)=-\frac{6 \mathcal{D}(y)}{\mathcal{T}(y)}\left(1-\frac{\gamma+1}{2 \gamma} \frac{\partial \ln j}{\partial \ln T}\right)-\frac{\partial \ln j}{\partial \ln L}-\frac{\mathcal{Y}(y)}{4 \mathcal{T}(y)} \frac{1-x^{2}}{x^{2}} \frac{\partial \ln j}{\partial \ln x}
$$

Where $j$ is measured at $t^{-}, x=\sqrt{1-y^{2}}$ is the cosine of the equatorial pitch angle, and $\mathcal{Y}$ is Schulz and Lanzerotti (1974)'s dipole bounce function (see also Lejosne and Mozer, 2020b for a detailed derivation of Eq 4). The main assumptions underlying this equation are that:

- There is no significant source or loss mechanism on the time scale of many hours (i.e. multiple drift periods), so that the phase space density $f=j / p^{2}$ is constant along the continuous trajectories of the system in phase space (Liouville's theorem);

- The timescale for electric field variations is such that the drift motion is the only perturbed motion: in other words, the first and second adiabatic invariants are conserved;

- There is no time variation of the magnetic field and the magnetic field is mainly dipolar.

\subsection{Data Set and Data Processing}

\subsubsection{Van Allen Probes Data Set}

We analyze directional differential fluxes between $L=1.5$ and $L=3$ in the $50-400 \mathrm{keV}$ energy range provided by the Radiation Belt Storm Probes Ion Composition Experiment (RBSPICE) instruments (Mitchell et al., 2013) onboard the two Van Allen Probes. The data products (level 3 Pitch Angle and Pressure High-Energy resolution, Low Time resolution, Electron Species Rates, lev-3-PAP_ESRHELT) offer high energy resolution (dE/E $\sim 0.065$ ) over 17 pitch angle channels with a $10^{\circ}$ resolution in almost every bin $\left(15^{\circ}\right.$ in the first and last bins).

The Van Allen Probes had a perigee at $\sim 600 \mathrm{~km}$, an apogee at $\sim 5.8 R_{E}$, a period of $\sim 9 \mathrm{~h}$ and an inclination of $\sim 10^{\circ}$. They crossed the inner belt and slot region multiple times per day, providing consecutive observations of the time evolution of the zebra stripes close to the magnetic equator. At the beginning of the year 2014, the inbound crossings of the region of interest $(1.5<\mathrm{L}<3)$ were in the dusk sector (16-19.5 MLT) while the outbound crossings were in the dawn sector (5-8 MLT).

\subsubsection{Data Processing}

To compute the fluctuations in directional differential fluxes, $\delta j$, it is necessary to determine the differential flux before the occurrence of electric field disturbances, $j\left(t^{-}\right)$(Eq 4). Because the event occurs at the beginning of 16 February 2014, we analyzed Van Allen Probes data during 15 February 2014. While fluxes of trapped energetic electrons have been shown to vary with magnetic local time due to drift shell distortion by the quiet time wind dynamo (e.g., Selesnick et al., 2016; Lejosne et al., 2021), this effect is found to be unimportant for the range of $\mathrm{L}$ values $(1.5<\mathrm{L}<3)$ and kinetic 


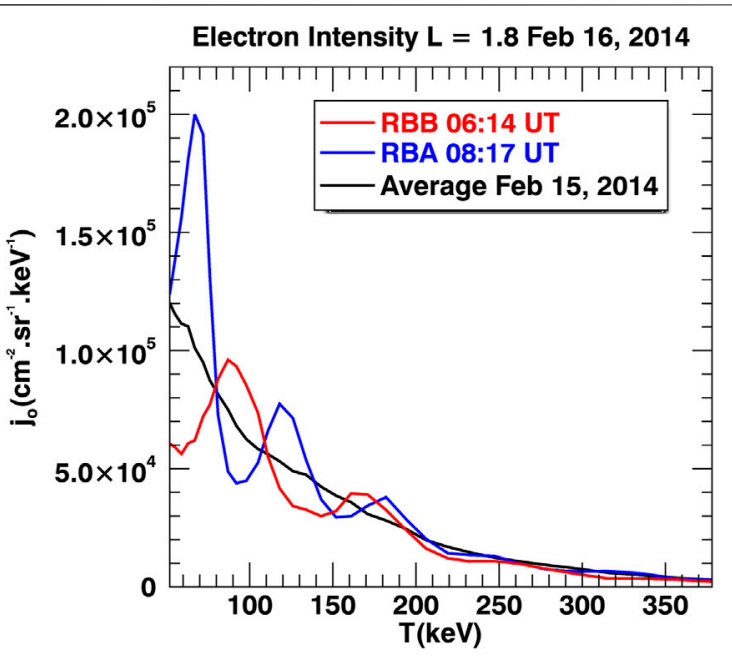

FIGURE 2 | Zebra stripes observed during two successive inbound crossings of $L=1.8$ (18 MLT), at 06:14 UT by Van Allen Probes B (RBB, in red) and at 08:17 UT by Van Allen Probes A (RBA, in blue). The time-varying equatorial electron intensity is compared with the average equatorial intensity at $L=1.8$ of 15 February 2014 (in black) and shows 100\% fluctuations in equatorial electron intensity at low energies ( 70 keV). energy (50-400 keV) considered in this study: $\Delta j / j$, the relative difference between equatorial fluxes measured in the dawn and dusk sectors of the same $\mathrm{L}$ value, decreases with $\mathrm{L}$ value and kinetic energy. It is less than $10 \%$ on average at $\mathrm{L}=1.5$, and less than $5 \%$ on average at $\mathrm{L}=1.7$. This simplifies the analysis as the magnetic local time dimension does not need to be taken into account when determining the differential flux before the occurrence of electric field disturbances, $j\left(t^{-}\right)$. For every $L$ value (+/- 0.05) between 1.5 and 3, and for each energy channel between 50 and $400 \mathrm{keV}$, the directional differential fluxes measured are represented as a function of the sine of the equatorial pitch angle, $y$. A linear least square regression is performed for the logarithm of the flux, $j$, as a function of the logarithm of the sine of the equatorial pitch angle, $y$, to find the best fitting sets of parameters $\left(\overline{j_{o}}, n\right)$ so that $j\left(T, y, L ; t^{-}\right) \cong \overline{j_{o}} y^{n} \equiv \bar{j}$. The error of the estimate is quantified by the root mean square error in every pitch angle bin $(x+1-0.05)$. Because the energetic electron fluxes did not vary significantly with time and magnetic local time on 15 February 2014, the fitting of the fluxes is relatively good: The error is of the order of $10 \%$ for near equatorial particles, and it increases with decreasing pitch angle - up to $50+\%$ for the most field aligned particles. Information on the fitting parameters $\left(\overline{j_{o}}, n\right)$ is then leveraged to estimate the function $\psi(T, y, L)(\mathbf{E q} 5)$. The partial derivatives $\partial \ln j / \partial \ln T, \partial \ln j / \partial \ln L$ and $\partial \ln j / \partial \ln x$ are estimated locally by computing the slopes of the linear fits between $\ln \bar{j}$ and $\ln T, \ln L$ and $\ln x$ in bins of size $0.1 L, 0.12 T$ and $0.1 x$, respectively.

Figure 2 shows the equatorial electron intensity at $\mathrm{L}=1.8$ during two successive inbound crossings on 16 February 2014 at 06:14 UT for Van Allen Probes B (18:14 MLT) and at 08:17 UT for Van Allen Probes A (18:23 MLT). The reference equatorial electron intensity, $\overline{j_{o}}$, is superimposed. The comparison between the different curves shows the presence of time-varying, energydependent fluctuations in the equatorial electron intensity, $\delta j / j$, up to $100 \%$ for $\sim 70 \mathrm{keV}$ electrons.

\section{RESULTS}

\subsection{Radial Transport From the Analysis of the Zebra Stripes}

Figure 3 illustrates how (Section 2.3) information on trapped particles' radial transport can be retrieved from an analysis of fluctuations in directional differential fluxes observed at a later time. The fluxes are similar to the ones shown in Figure 2. The fluctuations (Figure 3A) are divided by the function $\psi(T, y, L)$ to provide information on radial transport (Figure 3B). The oscillations in kinetic energy arise because the radial transport magnitude varies with the drift phase (i.e., magnetic local time) at

A Electron Intensity Fluctuation $L=1.8$ Feb 16, 2014

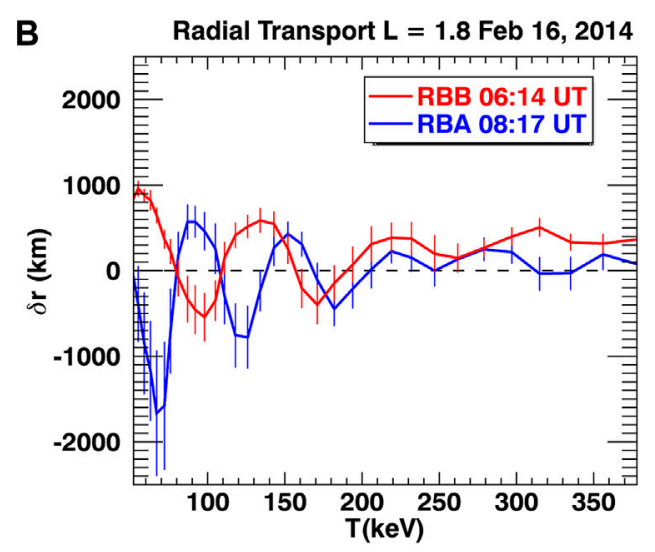

FIGURE 3 | (A) Electron intensity fluctuations, $\delta j / j$, in the $90^{\circ}$ pitch angle channel at $\mathrm{L}=1.8$ on 16 February 2014 at 06:14 UT according to Van Allen Probes B (RBB, in red) and at 08:17 UT according to Van Allen Probes A (RBA, in blue) and (B) corresponding radial transport in $\mathrm{km}, \delta r=R_{E} d L$. The error bars are mainly due to the uncertainty in the magnitude of the flux before the occurrence of the electric field disturbances, $j\left(t^{-}\right)$. 

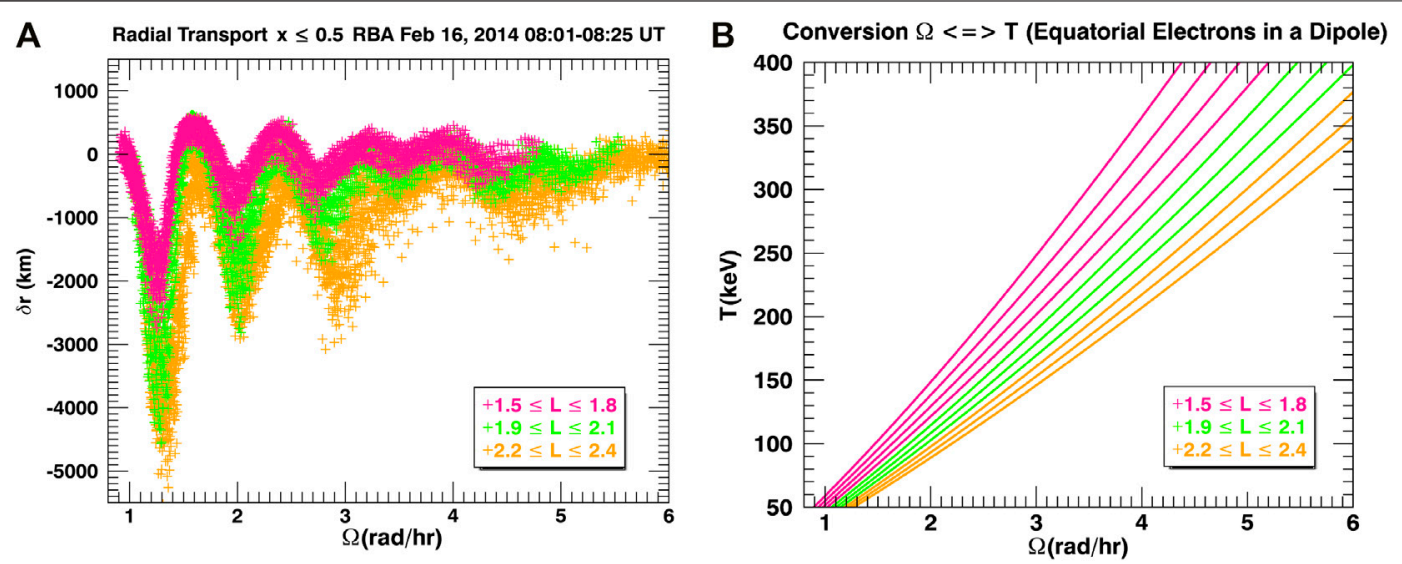

FIGURE 4 | (A) Radial transport, $\delta r=R_{E} d L$, caused by the electric field disturbances at the beginning of 16 February 2014 and estimated from an analysis of fluctuations in electron intensity (50-400 keV, equatorial pitch angle of $90^{\circ}+/-30^{\circ}$ ) measured by Van Allen Probes $A 7$ hours after the event. Three $L$ regions between $L=$ 1.5 and $L=2.4$ are represented by three colors to highlight the increase of radial transport with $L(1.5 \leq L \leq 1.8$ is in pink, $1.9 \leq L \leq 2.1$ is in green and $2.2 \leq L \leq 2.4$ is in orange). (B) Conversion between angular drift frequency and kinetic energy in the case of equatorial electrons trapped at various $L$ values (1.5 $<L<2.5)$ in a dipole field.

the time of the event, $t_{i}$, and this magnetic local time, $\varphi\left(t_{i}\right)$, is a function of kinetic energy: $\varphi\left(t_{i}\right)=\varphi-\Omega\left(t-t_{i}\right)$, where $\varphi$ is the magnetic local time of the observation at time, $t$, and $\Omega$ is the angular drift frequency (Eq. 1). Figure 3B suggests that the electric field disturbances generated radial transport of the order a thousand kilometers peak-to-peak at $L=1.8$ $(d L / L \sim 0.1)$. This is at least one order of magnitude greater than the effect of the quiet time wind dynamo, that generates radial distortion of the drift shell between dawn and dusk of the order of several tens of kilometers at $L=1.3$ (Section 2.1.2, see also Lejosne et al., 2021; Selesnick et al., 2016).

The approach illustrated in Figure 3 is applied to all inbound and outbound crossings of the inner belt and slot region during the morning of 16 February 2014. Figure 4A shows how radial transport generated multiple $(\sim 7)$ hours before observations is determined from an analysis of fluxes measured during one crossing of $1.5<\mathrm{L}<2.5$. The illustration is for trapped particles with a cosine of the equatorial pitch angle, $x$, below 0.5 (equatorial pitch angles between $60^{\circ}$ and $120^{\circ}$ ) and below $L=2.5$, to focus on estimates with relatively small error bars. Figure $4 B$ provides the kinetic energy, $T$, associated with the angular drift frequency, $\Omega$, in the case of equatorial electrons trapped at various $L$ values $(1.5<L<2.5)$ in a dipole field (Eq. 1 and Eq. 2).

Figure 4A shows how radial transport oscillates with angular drift frequency, $\Omega$ (Eq. 1 and Eq. 2) and increases with increasing $L$ values. These findings are consistent with the picture in which the prompt penetration of high latitude electric fields quickly modifies trapped particles' drift motion, coherently over a large $(>1 \mathrm{~L})$ portion of the inner belt and slot region, and with an effect that depends on the trapped particles' location at the time of the event. The oscillation with angular drift frequency indicates that the amount of radial transport depends on the initial magnetic local time of the electrons in the disturbance. Indeed, the angular drift frequencies that correspond to the same phase of the periodic signal share the same initial magnetic local time, $\varphi\left(t_{i}\right)$. The increase with $L$ values indicates that radial transport is most efficient at higher $L$ values. We emphasize that radial transport is not expected to depend on pitch angle in the electrostatic case (e.g., Fälthammar, 1968).

The radial transport estimates shown in Figure 4 as a function of the angular drift frequency do not oscillate symmetrically around a value of zero: They are negative (i.e. inward) on average. One possible interpretation of this observation is that the drift motion of the trapped particles has also been altered by a slower (occurring on a time scale greater than trapped particles' drift periods) variation of the electric fields. A slow inward transport of the trapped particles requires a relatively steady westward component for the time varying electric fields (Eq. 3). This could be due to the disturbance dynamo action of the storm time winds for instance.

We further investigate the dependence of radial transport with equatorial radial distance, $L$, by performing a series of linear least

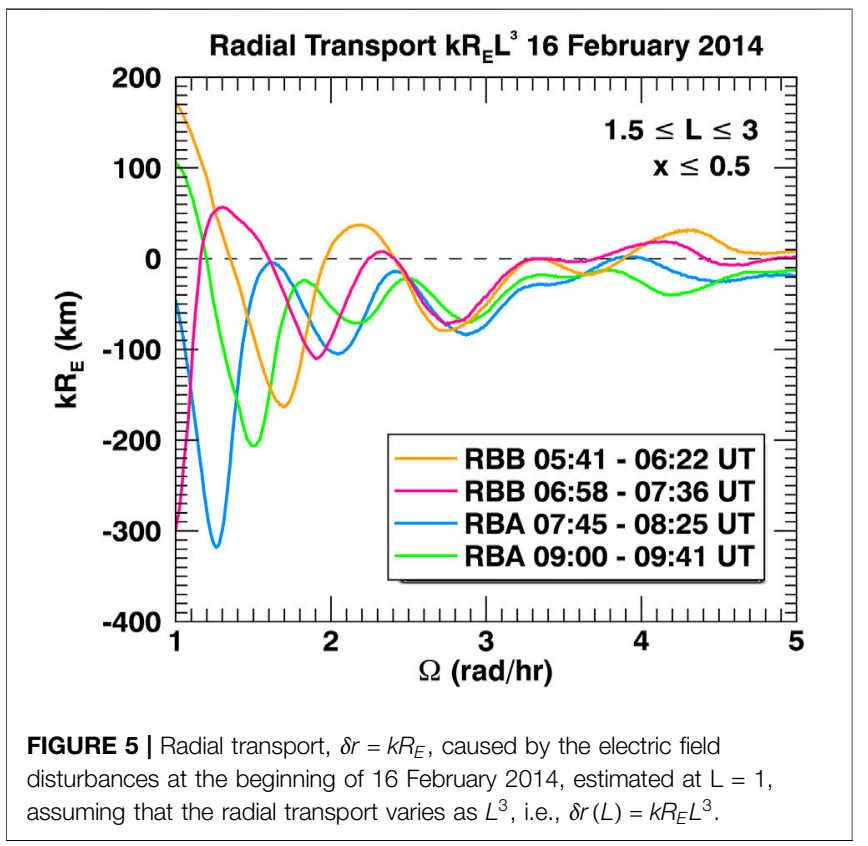



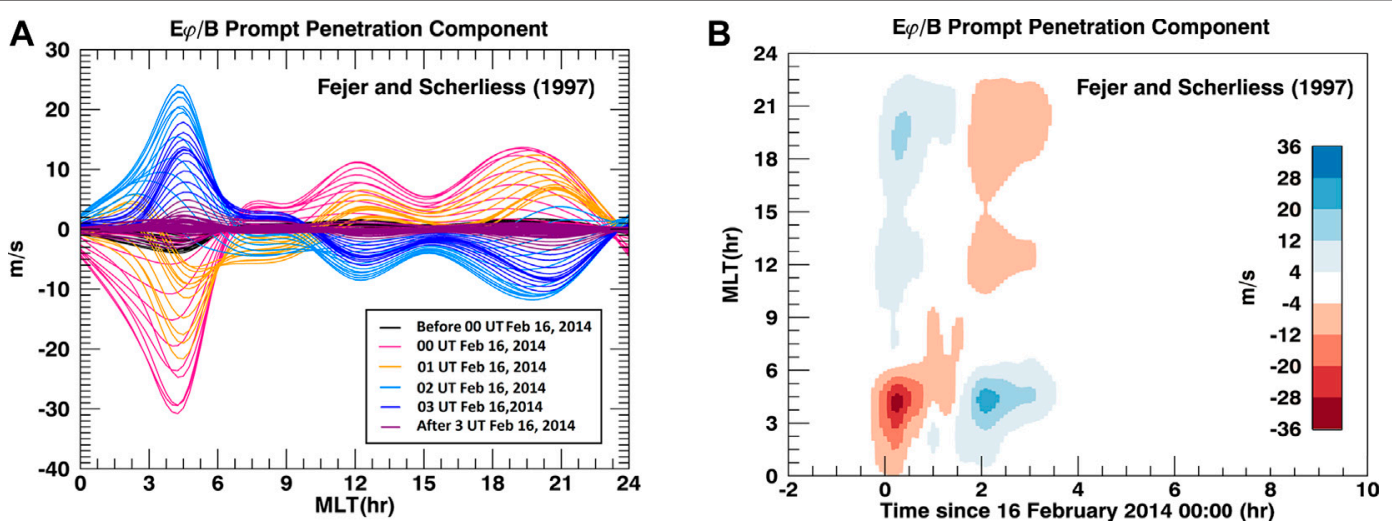

FIGURE 6 | 12 h of radial drift motion driven by the prompt penetration of magnetospheric convection between 15 February 2014,22 UT and 16 February 2014,10 UT, according to the analytical empirical model developed by Fejer and Scherliess (1997). (A) The amplitude is represented as a function of magnetic local time, MLT, for different times. (B) The amplitude is color-coded as a function of the number of hours since 16 February 2014,00 UT and the magnetic local time, MLT.

square regressions. For every angular drift frequency, $\Omega(+/-0.1 \mathrm{rad} / \mathrm{hr})$, and for every crossings of the inner belt and slot region $(1.5<\mathrm{L}<3)$, we collect information on average radial transport as a function of $L(+/-0.1)$. Because radial transport is not expected to depend on pitch angle in the electrostatic case (e.g., Fälthammar, 1968), we collect all data points relative to trapped particles with equatorial pitch angle of $90^{\circ}+1-30^{\circ}(x \leq 0.5)$. Because radial transport is expected to vary as a power of $L, \delta r=k R_{E} L^{n}$, we perform linear least square regressions between $\ln |\delta r|$ and $\ln L$ to determine the best sets of $k$ and $n$ parameters for each angular drift frequency and inner belt crossing time. We find estimates for the power of $L$ associated with radial transport, $n$, that are variable when considering all inbound and outbound crossings of the inner belt and slot region during the morning of 16 February 2014. The median value is of the order of $n=3$, with an interquartile range of 3 (value of the first quartile: 2; value of the third quartile: 5 ). Considering that radial transport is proportional to $L^{3}$ is equivalent to assuming that the electric field disturbances is uniform in the inner belt and slot region (Eq. 3), a finding seemingly consistent with the prompt penetration of magnetospheric convection.

The estimates for the $k$ parameters are obtained by minimizing the least squares of $\delta r / R_{E} L^{3}$. The results, provided in Figure 5, correspond to radial transport extrapolated to $\mathrm{L}=1$. It is of the order of $300 \mathrm{~km}$ peak-to-peak for $\Omega \sim 1 \mathrm{rad} / \mathrm{hr}$ and decreases to a few tens of kilometers for $\Omega \geq 3 \mathrm{rad} / \mathrm{hr}$. Decreasing (resp. increasing) the assumed power of $L$ by one unit in the least square fit $\left(n^{\prime}=n \mp 1\right)$ increases (resp. decreases) the resulting peak-to-peak amplitude of radial transport at $\mathrm{L}=1$ by factor $\sim 2$.

\subsection{Radial Transport at $L=1$ From Particle Tracing}

We apply the model developed by Fejer and Scherliess to compute the perturbation in radial motion (i.e., the ionospheric meridional drift disturbance) based on the time history of the AE index. According to the model, the prompt penetration electric fields are by far the main contributors to the perturbation in radial motion (Section 2.2). The spatial and temporal variations of the electric drift associated with prompt penetration of magnetospheric dynamo electric field is represented in Figure 6. The time interval is from 15 February 2014, 22 UT to 16 February 2014, 10 UT. The sharp increase in the AE index at the beginning of 16 February 2014 is associated with a strong inward motion around 5 MLT (with a maximum magnitude of $\sim-30 \mathrm{~m} / \mathrm{s}$ ). Similarly, the sharp decrease in $\mathrm{AE}$ index around two UT is associated with a strong outward motion around 5 MLT (with a maximum magnitude of $24 \mathrm{~m} / \mathrm{s}$, i.e., $0.6 \mathrm{mV} / \mathrm{m}$ ). In comparison, the perturbation in radial motion velocity associated with the disturbance dynamo remains smaller than $6 \mathrm{~m} / \mathrm{s}(0.15 \mathrm{mV} / \mathrm{m})$ throughout the same time interval. With this model, the characteristic time for the variation of the fields is shorter than the drift period ( $<1 \mathrm{~h}$ vs. $1.25 \mathrm{hr}-6.3 \mathrm{hr}$ for $\Omega$ between $1 \mathrm{rad} / \mathrm{hr}$ and $5 \mathrm{rad} / \mathrm{hr}$ ).

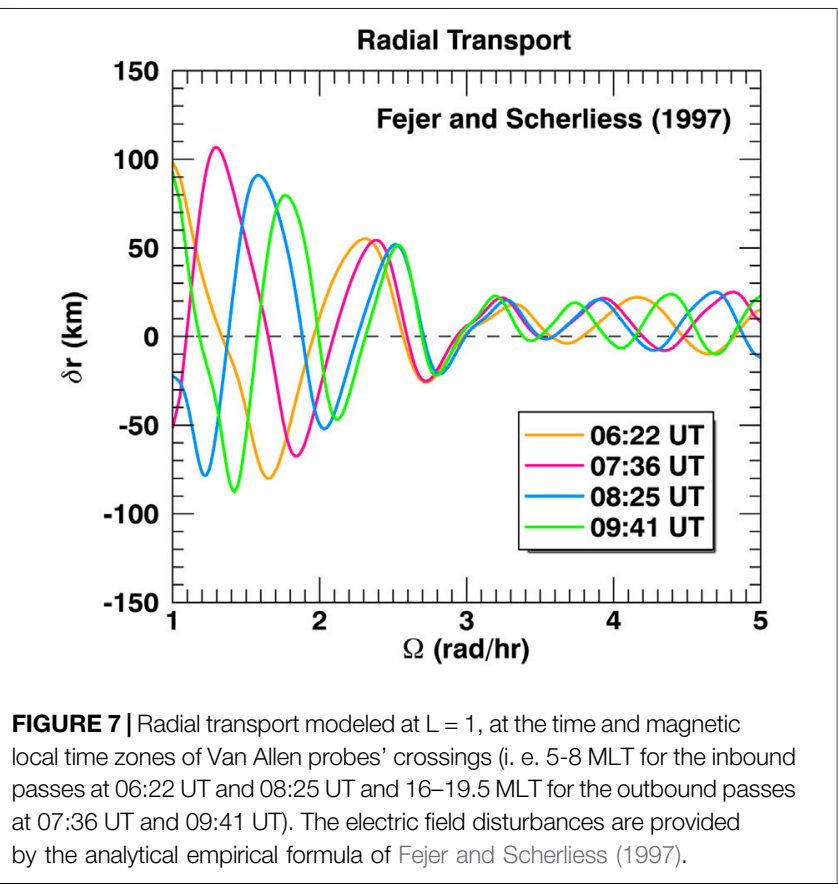


We use these outputs to solve the dynamics of particles unrealistically trapped at $\mathrm{L}=1$. Using Eq. 3, we launch particles with different angular drift velocities, $\Omega$, and different drift phases at L $=1$ on 15 February 2014, 22 UT. At the time of the end of every crossing (06:22 UT, 07:36 UT, 08:25 UT and 09: $41 \mathrm{UT})$, we select the particles that are in the same magnetic local time zone as the one scanned by the Van Allen Probes and we store information relative to their radial transport, $\delta r$. The results are provided in Figure 7.

The radial transport computed using the modeled electric field disturbances (prompt penetration and disturbance wind dynamo) varies as expected with time, magnetic local time, and angular drift velocity, $\Omega$. It is of the order of $150 \mathrm{~km}$ peak-to-peak for $\Omega \sim 1-2 \mathrm{rad} / \mathrm{hr}$, and decreases to a few tens of kilometers for $\Omega \geq 3 \mathrm{rad} / \mathrm{hr}$.

\subsection{Model-Observation Comparison}

The comparison of Figure 5 and Figure 7 shows that the local minima and maxima seen in the experimental estimates for radial transport correspond to the ones obtained numerically. The Pearson correlation coefficient, $c c$, between the two series (experimental and numerical estimates of radial transport) is high during the first pass, and then decreases with time (06: 22UT $c c=0.76$; 07:36 UT $c c=0.52$; 08:25 UT cc $=0.49$; 09:41 $\mathrm{UT} \mathrm{cc}=0.39)$. The good agreement between the locations of the extrema and the similitude in the overall shapes of the envelopes suggest that the timing for electric field variations provided by the model is valid for studying radial transport in the inner belt and slot region. On the other hand, the amount of radial transport provided by the model (Figure 7) is smaller than the one resulting from the extrapolation of radial transport experimental estimates down to $\mathrm{L}=1$ (Figure 5), by

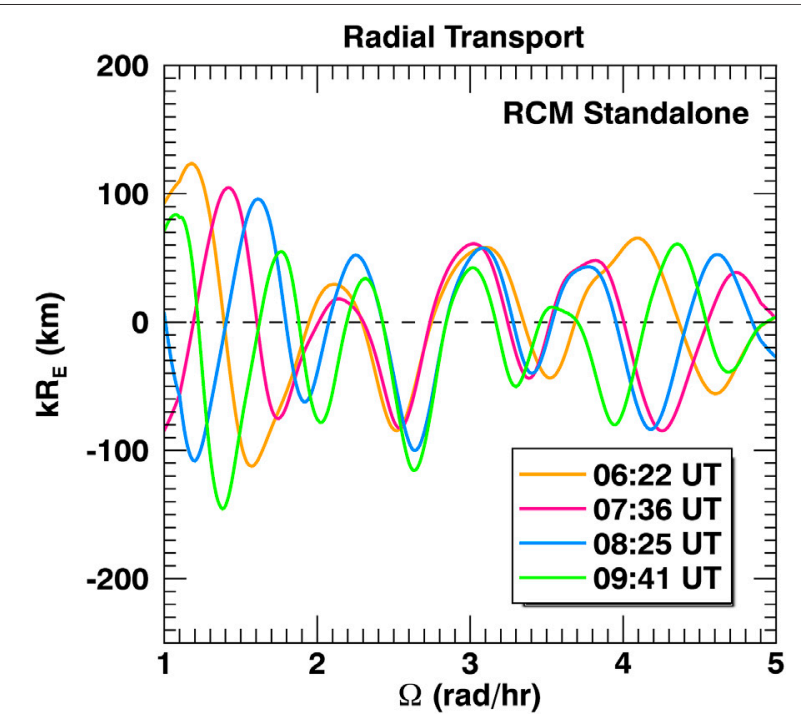

FIGURE 8 | Radial transport modeled at $L \sim 1$, at the time and magnetic local time zones of Van Allen probes' crossings (i. e. 5-8 MLT for the inbound passes at 06:22 UT and 08:25 UT and 16-19.5 MLT for the outbound passes at 07:36 UT and 09:41 UT). The electric field disturbances are provided by the standalone version of $R C M$ at $L=1.03$. approximately a factor 2-3. Because the numerical model comes from an average over many scenarios, it is possible that the penetration electric fields are underestimated in this case. Another difference is that the experimental estimates for radial transport are predominantly negative while the numerical estimates oscillate around zero. This negative offset is present during both inbound passes in the dusk sector and outbound passes in the dawn sector. This rules off the quiet time wind dynamo as the cause of the offset, since the effect of the quiet time wind dynamo depends on local time. Even though the effect of the electric fields due to the disturbance wind dynamo are taken into account in the particle tracing, this discrepancy suggests that their effect is greater than predictions. It could be that the effect of the dynamo electric fields increases with equatorial radial distance in the magnetosphere.

\section{DISCUSSION}

\subsection{Comparing With Radial Transport Based on the Electric Field Dynamics Provided by the Rice Convection Model}

We further investigate the model-data discrepancies by running the University of Colorado's version of the standalone Rice Convection Model (RCM) for 15-16 February 2014 (e.g, Maruyama et al., 2011). The data gaps in the OMNI solar wind parameters are filled using WIND measurements. The modeled prompt penetration electric fields are provided at $100 \mathrm{~km}$ altitude, and they are mapped to the magnetic equator assuming equipotential field lines in a magnetic dipole (e.g., Lejosne and Mozer, 2016). Specifically, for the azimuthal electric field component: $E_{\varphi}=E_{\varphi \text {,iono }} L^{-3 / 2}$, where $E_{\varphi \text {,iono }}$ is the ionospheric eastward electric field. The time resolution for model outputs is $10 \mathrm{~min}$. The magnitude of the peak inward motion increases from a value of $-65 \mathrm{~m} / \mathrm{s}$ at 04:30 MLT around 00 UT on 16 February 2014 to a value of up to $38 \mathrm{~m} / \mathrm{s}$ at 00:20 UT at 03:15 MLT. These magnitudes are $\sim 2$ times larger than the extrema provided by the analytical empirical model (Figure 6A). In addition, the electric field variations occur on a shorter timescale with RCM $(+100 \mathrm{~m} / \mathrm{s}$ within $20 \mathrm{~min}$ for RCM versus $+50 \mathrm{~m} / \mathrm{s}$ within $2 \mathrm{~h}$ for the analytical empirical model). The results of the particle tracing at $\mathrm{L}=1 \mathrm{using}$ RCM electric field disturbances are presented in Figure 8.

The quality of the agreement between model and data is lesser here than in Section 3.3, with correlation coefficients below 0.5 in all passes $(06: 22 \mathrm{UT} \mathrm{cc}=0.38 ; 07: 36 \mathrm{UT} \mathrm{cc}=0.35 ; 08: 25 \mathrm{UT} \mathrm{cc}=$ $0.42 ; 09: 41 \mathrm{UT} \mathrm{cc}=0.14)$. That the amplitude of radial transport does not decrease with the angular drift frequency indicates that the characteristic timescale for the variations of the field is faster than the drift period in all cases. This is in contrast with the experimental result, where radial transport decreases and reaches values of the order of a few tens of kilometers for $\Omega \geq 3 \mathrm{rad} / \mathrm{hr}$ (Figure 5). This discrepancy suggests that the electric fields vary too rapidly in the simulation.

We leverage the fact that RCM informs on electric field dynamics at all magnetic latitudes to determine the power of $\mathrm{L}, n$, associated with radial transport in the simulations. The 
average value is of the order of $n=3$, with an interquartile range of 0.6, in agreement with results from the experimental data analysis (Section 3.1).

\subsection{Other Considerations}

\subsubsection{Instrument Resolution}

An instrument with a high-energy resolution is required when it comes to resolving and analyzing drift echoes such as the zebra stripes (e.g., Hartinger et al., 2018). Particles with slightly different kinetic energies sharing the same energy channel will have mixed adiabatically on a time scale of the order of $2 \pi / d \Omega$ (Schulz and Lanzerotti, 1974), i.e. of the order of $2 \pi /(\Omega(\mathrm{dE} / \mathrm{E}))$. This effect can preclude the application of the method introduced in Section 2.3 when the time interval between the event and the observation of the resulting flux variations is too long. The resolution of the RBSPICE instruments is $\mathrm{dE} / \mathrm{E}$ of $\sim 0.06$. Thus, for trapped particles with angular drift velocities of $5 \mathrm{rad} / \mathrm{hr}$, the timescale for adiabatic mixing is $\sim 20 \mathrm{~h}$. That said, damping in the amplitude of the zebra stripes occurs on shorter timescales. For instance, the zebra stripes observed in the afternoon of 16 February 2014 have significantly smaller peak-to-peak amplitudes than the ones observed during the morning. That is why they were omitted from this study.

\subsubsection{Radial Diffusion in the Inner Belt and Slot Region} The power of $L$ associated with radial transport informs the power of $L$ associated with radial diffusion, since $D_{L L}=\left[(\Delta L)^{2}\right] / 2 \Delta t$, where $\Delta L$ corresponds to the normalized radial transport after a time $\Delta t$. This analysis suggests that radial diffusion associated with the prompt penetration of magnetospheric convection has a power of $L$ equal to 6 in the inner belt and slot region. A statistical analysis of zebra stripe events is necessary to determine the frequency and average magnitude of radial transport, both of which are needed to quantify radial diffusion driven by magnetic activity below $\mathrm{L}=3$.

\section{REFERENCES}

Brand, A., Allen, L., Altman, M., Hlava, M., and Scott, J. (2015). Beyond Authorship: Attribution, Contribution, Collaboration, and Credit. Learn. Pub. 28, 151-155. doi:10.1087/20150211

Fälthammar, C.-G. (1965). Effects of Time-Dependent Electric fields on Geomagnetically Trapped Radiation. J. Geophys. Res. 70 (11), 2503-2516. doi:10.1029/JZ070i011p02503

Fälthammar, C.-G. (1968). "Radial Diffusion by Violation of the Third Adiabatic Invariant," in Earth's Particles and fields. Editor B. M. McCormac (New York: Reinhold), 157-169.

Fejer, B. G., and Scherliess, L. (1995). Time Dependent Response of Equatorial Ionospheric Electric fields to Magnetospheric Disturbances. Geophys. Res. Lett. 22 (7), 851-854. doi:10.1029/95GL00390

Fejer, B. G., and Scherliess, L. (1997). Empirical Models of Storm Time Equatorial Zonal Electric fields. J. Geophys. Res. 102 (A11), 24047-24056. doi:10.1029/ 97JA02164

Fejer, B. G., Blanc, M., and Richmond, A. D. (2017). Post-Storm Middle and LowLatitude Ionospheric Electric Fields Effects. Space Sci. Rev. 206, 407-429. doi:10. 1007/s11214-016-0320-x

\section{DATA AVAILABILITY STATEMENT}

Publicly available datasets were analyzed in this study. This data can be found here: http://rbspice.ftecs.com/Data.html (RBSPICE database). Simulation outputs can be obtained by contacting NM.

\section{AUTHOR CONTRIBUTIONS}

The first author is the lead and corresponding author. All other authors are listed in alphabetical order. We describe contributions to the paper using the CRediT (Contributor Roles Taxonomy) categories (Brand et al., 2015). Conceptualization, Formal Analysis, Writing-Original Draft, Visualization and Project Administration: SL Funding acquisition, Methodology: SL and NM Resources, Software: SL, BF, NM, and LS. Investigation: SL, BF and LS. Writing-Review \& Editing: SL, BF, NM and LS.

\section{FUNDING}

SL work was performed under NASA Grant Awards 80NSSC18K1223 and 80 NSSC20K1351. The work of BF was supported by the NASA H-LWS program through grant 80NSSC17K71.

\section{ACKNOWLEDGMENTS}

The use of OMNI data and NASA/GSFC's Space Physics Data Facility's OMNIWeb (https://omniweb.gsfc.nasa.gov/) service is acknowledged. We also acknowledge the substorm timing list identified by the Newell and Gjerloev technique (Newell and Gjerloev, 2011a), the SMU and SML indices (Newell and Gjerloev, 2011b); and the SuperMAG collaboration (Gjerloev, 2012). SL thanks C. Huang for a helpful discussion about ionospheric E-field measurements. SL and NM thank Mariangel Fedrizzi for a helpful discussion about filling data gaps in solar wind measurements.

Gjerloev, J. W. (2012). The SuperMAG Data Processing Technique. J. Geophys. Res. 117, A09213. doi:10.1029/2012JA017683

Hartinger, M. D., Claudepierre, S. G., Turner, D. L., Reeves, G. D., Breneman, A., Mann, I. R., et al. (2018). Diagnosis of ULF Wave-Particle Interactions with Megaelectron Volt Electrons: The Importance of Ultrahigh-Resolution Energy Channels. Geophys. Res. Lett. 45, 11,883-11,892. doi:10.1029/2018GL080291

Imhof, W. L., and Smith, R. V. (1965). Observation of Nearly Monoenergetic HighEnergy Electrons in the Inner Radiation Belt. Phys. Rev. Lett. 14, 885-887. doi:10.1103/PhysRevLett.14.885

Lanzerotti, L. J., Roberts, C. S., and Brown, W. L. (1967). Temporal Variations in the Electron Flux at Synchronous Altitudes. J. Geophys. Res. 72 (23), 5893-5902. doi:10.1029/JZ072i023p05893

Lejosne, S., and Mozer, F. S. (2016). Typical Values of the Electric Drift E $\times$ B/B 2 in the Inner Radiation belt and Slot Region as Determined from Van Allen Probe Measurements. J. Geophys. Res. Space Phys. 121, 12014-12024. doi:10.1002/ 2016JA023613

Lejosne, S., and Mozer, F. S. (2018). Magnetic Activity Dependence of the Electric Drift below L $=3$. Geophys. Res. Lett. 45, 3775-3782. doi:10.1029/ 2018GL077873

Lejosne, S., and Mozer, F. S. (2020a). Experimental Determination of the Conditions Associated with "Zebra Stripe" Pattern Generation in the Earth's 
Inner Radiation Belt and Slot Region. J. Geophys. Res. Space Phys. 125, e2020JA027889. doi:10.1029/2020JA027889

Lejosne, S., and Mozer, F. S. (2020b). Inversion of the Energetic Electron "Zebra Stripe" Pattern Present in the Earth's Inner Belt and Slot Region: First Observations and Interpretation. Geophys. Res. Lett. 47, e2020GL088564. doi:10.1029/2020GL088564

Lejosne, S., and Roederer, J. G. (2016). The "Zebra Stripes”: An Effect of $F$ Region Zonal Plasma Drifts on the Longitudinal Distribution of Radiation belt Particles. J. Geophys. Res. Space Phys. 121, 507-518. doi:10.1002/2015JA021925

Lejosne, S., Fedrizzi, M., Maruyama, N., and Selesnick, R. S. (2021). Thermospheric Neutral Winds as the Cause of Drift Shell Distortion in Earth's Inner Radiation Belt. Front. Astron. Space Sci. 8, 725800. doi:10.3389/fspas.2021.725800

Liu, Y., Zong, Q.-G., Zhou, X.-Z., Foster, J. C., and Rankin, R. (2016). Structure and Evolution of Electron "Zebra Stripes" in the Inner Radiation belt. J. Geophys. Res. Space Phys. 121, 4145-4157. doi:10.1002/2015JA022077

Maruyama, N., Fuller-Rowell, T. J., Codrescu, M. V., Anderson, D., Richmond, A. D., Maute, A., et al. (2011). "Modeling the Storm Time Electrodynamics," in Aeronomy of the Earth's Atmosphere and Ionosphere, IAGA Special Sopron Book Series. Editors M. Abdu and D. Pancheva (Dordrecht: Springer), 2, 455-464. doi:10.1007/978-94-007-0326-1_35

Mauk, B. H., Fox, N. J., Kanekal, S. G., Kessel, R. L., Sibeck, D. G., and Ukhorskiy, A. (2013). Science Objectives and Rationale for the Radiation Belt Storm Probes Mission. Space Sci. Rev. 179, 3-27. doi:10.1007/s11214-012-9908-y

Mitchell, D. G., Lanzerotti, L. J., Kim, C. K., Stokes, M., Ho, G., Cooper, S., et al. (2013). Radiation Belt Storm Probes Ion Composition Experiment (RBSPICE). Space Sci. Rev. 179, 263-308. doi:10.1007/s11214-013-9965-x

Mozer, F. S. (1970). Electric Field Mapping in the Ionosphere at the Equatorial Plane. Planet. Space Sci. 18, 259-263. doi:10.1016/0032-0633(70)90161-3

Newell, P. T., and Gjerloev, J. W. (2011a). Evaluation of SuperMAG Auroral Electrojet Indices as Indicators of Substorms and Auroral Power. J. Geophys. Res. 116, A12211. doi:10.1029/2011JA016779

Newell, P. T., and Gjerloev, J. W. (2011b). Substorm and Magnetosphere Characteristic Scales Inferred from the SuperMAG Auroral Electrojet Indices. J. Geophys. Res. 116, A12232. doi:10.1029/2011JA016936

Richmond, A. D., Blanc, M., Emery, B. A., Wand, R. H., Fejer, B. G., Woodman, R. F., et al. (1980). An Empirical Model of Quiet-Day Ionospheric Electric Fields at Middle and Low Latitudes. J. Geophys. Res. 85, 4658-4664. doi:10.1029/ JA085iA09p04658

Roederer, J. G., Hilton, H. H., and Schulz, M. (1973). Drift Shell Splitting by Internal Geomagnetic Multipoles. J. Geophys. Res. 78 (1), 133-144. doi:10.1029/ JA078i001p00133
Sandel, B. R., Goldstein, J., Gallagher, D. L., and Spasojevic, M. (2003). Extreme Ultraviolet Imager Observations of the Structure and Dynamics of the Plasmasphere. Space Sci. Rev. 109, 25-46. doi:10.1023/b:spac.0000007511. $47727.5 \mathrm{~b}$

Sauvaud, J.-A., Walt, M., Delcourt, D., Benoist, C., Penou, E., Chen, Y., et al. (2013). Inner Radiation belt Particle Acceleration and Energy Structuring by Drift Resonance with ULF Waves during Geomagnetic Storms. J. Geophys. Res. Space Phys. 118, 1723-1736. doi:10.1002/jgra.50125

Schulz, M., and Lanzerotti, L. J. (1974). Particle Diffusion in the Radiation Belts. Berlin, Heidelberg: Springer-Verlag. doi:10.1007/978-3-642-65675-0

Selesnick, R. S., Su, Y. J., and Blake, J. B. (2016). Control of the Innermost Electron Radiation belt by Large-scale Electric fields. J. Geophys. Res. Space Phys. 121, 8417-8427. doi:10.1002/2016JA022973

Sun, Y. X., Roussos, E., Hao, Y. X., Zong, Q.-G., Liu, Y., Lejosne, S., et al. (2021). Saturn's Inner Magnetospheric Convection in the View of Zebra Stripe Patterns in Energetic Electron Spectra. J. Geophys. Res. Space Phys. 126, e2021JA029600. doi:10.1029/2021ja029600

Tsyganenko, N. A. (1989). A Magnetospheric Magnetic Field Model with a Warped Tail Current Sheet. Planet. Space Sci. 37 (1), 5-20. doi:10.1016/0032-0633(89) 90066-4

Ukhorskiy, A. Y., Sitnov, M. I., Mitchell, D. G., Takahashi, K., Lanzerotti, L. J., and Mauk, B. H. (2014). Rotationally Driven 'Zebra Stripes' in Earth's Inner Radiation belt. Nature 507, 338-340. doi:10.1038/nature13046

Conflict of Interest: The authors declare that the research was conducted in the absence of any commercial or financial relationships that could be construed as a potential conflict of interest.

Publisher's Note: All claims expressed in this article are solely those of the authors and do not necessarily represent those of their affiliated organizations, or those of the publisher, the editors and the reviewers. Any product that may be evaluated in this article, or claim that may be made by its manufacturer, is not guaranteed or endorsed by the publisher.

Copyright (c) 2022 Lejosne, Fejer, Maruyama and Scherliess. This is an open-access article distributed under the terms of the Creative Commons Attribution License (CC $B Y)$. The use, distribution or reproduction in other forums is permitted, provided the original author(s) and the copyright owner(s) are credited and that the original publication in this journal is cited, in accordance with accepted academic practice. No use, distribution or reproduction is permitted which does not comply with these terms. 